\title{
Operations Risk Management by Optimally Planning the Qualified Workforce Capacity
}

\author{
Emmanuel Fragnière $^{*} \quad$ Jacek Gondzio $^{\dagger} \quad$ Xi Yang ${ }^{\ddagger}$
}

March 17, 2008, revised February 12th, 2009

\begin{abstract}
Operational risks are defined as risks of human origin. Unlike financial risks that can be handled in a financial manner (e.g. insurances, savings, derivatives), the treatment of operational risks calls for a "managerial approach". Consequently, we propose a new way of dealing with operational risk, which relies on the well known aggregate planning model. To illustrate this idea, we have adapted this model to the case of a back office of a bank specializing in the trading of derivative products. Our contribution corresponds to several improvements applied to stochastic programming techniques. First, the model is transformed into a multistage stochastic program in order to take into account the randomness associated with the volume of transaction demand and with the capacity of work provided by qualified and non-qualified employees over the planning horizon. Second, as advocated by Basel II, we calculate the probability distribution based on a Bayesian Network to circumvent the difficulty of obtaining data which characterizes uncertainty in operations. Third, we go a step further by relaxing the traditional assumption in stochastic programming that imposes a strict independence between the decision variables and the random elements. Comparative results show that in general these improved stochastic programming models tend to allocate more human expertise in order to hedge operational risks. Finally, we employ the dual solutions of the stochastic programs to detect periods and nodes that are at risk in terms of the expertise availability.
\end{abstract}

\footnotetext{
${ }^{*}$ Haute Ecole de Gestion of Geneva and University of Bath, 7 route de Drize, 1227 Carouge, Switzerland, Emmanuel.Fragniere@hesge.ch

${ }^{\dagger}$ University of Edinburgh, JCMB, Kings Building, West Mains Road, UK, J.Gondzio@ed.ac.uk

${ }^{\ddagger}$ University of Edinburgh, JCMB, Kings Building, West Mains Road, UK, X.Yang@ed.ac.uk
} 


\section{Introduction}

Back offices of banks typically deal with the transactions of financial contracts and with all the related paperwork and database management. For example, a trader (front office) "writes" an over-the-counter option with a counterparty. The back office will prepare the contracts, conduct all the exchange of information in due time and comply at the same time with the very stringent financial regulations. In the more and more frequent cases where the back office deals with derivative products, workers have to understand complicated pricing systems because it is part of their duties to conduct some price settlements, "reconciliations" and verifications. Several surveys (see [1, 15]) also indicate that operations of back offices are becoming far more complicated than before. The reasons for this evolution are multiple: IT harmonization due to banks consolidation, new stringent norms and regulations affecting the operations (e.g. IAS-IFRS, Sarbannes-Oxley, Basel II, new taxation system like Qualified Intermediary), significant increase of service productivity over the last years, boom of new sophisticated (structured) financial products. So more and more complex risks are attached to the activities of back offices and most of them require above all knowledge, experience and expertise to be addressed correctly and in an efficient manner.

Unfortunately, the risk management of back offices in the banking sector has not benefited from the modeling advances in financial risks (market, credit and liquidity risks) promulgated by the Basel Committee (www.bis.org). Basel II classifies risks of a bank in four categories: strategic; financial; non-financial/operational; reputation and compliance risks. Regarding the categories of non-financial/operational risks, four subcategories are used: fraud, political, IT, operations (transactions mistakes, inefficiencies of processes) risks. In operations, risks have two facets. They can be internal and external. Internal risk is typically linked to operations (hence controllable). External risks are the consequence of external causes (for instance, a change of some US GAAP accounting rules for derivatives which will affect the back office procedures as well as the training of the staff).

The logic underlying risk management in a Basel II context is always the same for each category of banking risks. Basel II proposes two methods: the standardized and the advanced ones. When the bank invests in qualified staff, software, and develops an advanced model, the bank is able to "economize" some capital assuming that it contributes actively to risk management. When the bank is not up to developing its own advanced model, then the capital that is to be set aside is calculated using a standardized approach. So far, the modeling of operational risk has not been much developed. This task is rather subjective regarding its qualitative nature, being related to managerial issues. 
A notion arising from service science makes the distinction between explicit (information) and implicit (or tacit) knowledge. Typically in a back office, tasks are very standardized and documented in working procedures. These tasks correspond to the explicit knowledge. Tacit knowledge on the other hand as defined by [22] corresponds to information that is difficult to express, formalize or share, in contrast to explicit knowledge, which is conscious and can be put into words. When something unexpected happens that might affect the service production, which is not included in the procedures, solely the expertise of qualified workers will permit correction of the problem and a return to normal. This statement is confirmed for instance in [23] that qualitative skills, like information search style, level of education and training on risk, influence the capability of risk manager to identify risks. In the case of operations, the risk can "materialize" under different states (see [7]) according to the TEID model: Threat, Event, Ignorance, Damage. Typically, the qualified worker knows how to act on these different states of risks through prevention, identification, and protective approaches.

In this paper we argue that the aggregate planning model adapted to services can be successfully applied to assess the level of expertise necessary to deal with operations risks in the back offices of banks. We demonstrate it using different extensions of the stochastic programming version of the aggregate planning model. The Aggregate Planning Model (APM) was developed in the middle of last century and has been successfully applied in production planning problems, see $[29,17,12]$, and manpower planning problems, see [21, 10, 2]. The most important feature of the aggregate planning model is the aggregation, either of products or manpower or both, which are presented as inventory constraints. The novelty of approach presented in this paper is to apply APM to services rather than to goods. Indeed, the "production" of services is intangible (see [26] for the characteristics of service). In other words, services do not produce goods and therefore cannot contain inventory of goods. In the APM approach presented in this paper, we consider the inventory of human expertise available in the back office. Starting from a classical multistage linear programming version of the aggregate planning model, we develop several extensions that enable us to capture the true nature of operations risks.

In a real context of enterprise risk management, when future events need to be considered in business activity planning, uncertainty of parameters plays the key role. Initiated in the late fifties by Dantzig and Madansky, Stochastic Programming provides a paradigm to include uncertainty into optimization-based decision models $[4,20]$. In particular, a multistage stochastic program with recourse is a multi-period mathematical program where parameters are assumed to be uncertain along the time path. The term recourse means that the deci- 
sion variables adapt to the different outcomes of random parameters at each time period. Stochastic programming model allows one to handle simultaneously several scenarios. It provides an adaptive policy that is close in spirit to the way decision-makers have to deal with uncertain futures in real life. A first improvement of the basic APM we explore to better manage operational risk is to assume that capacities as well as demand are uncertain, as will be shown in Section 3. In this context it corresponds to a situation where the risk is external (like a market risk) and we consider that the quality of work has no influence whatsoever on it (it is uncontrollable through managerial activities). The solution simply adapts to the evolution of the different scenarios described by the event tree.

A second improvement, as advocated by Basel II, is to calculate probability distribution based on Bayesian network. This reflects the observation that data related to operations risks are rarely available. We thus apply this scheme to our basic model where the demand parameters are assumed to be random. Bayesian method, which is similar to our method in the sense of probability calculation based on Bayes rule, has been applied for example by Morton and Popova [25] to evaluate capacity strategies of a manufacturing problem.

A third improvement to deal with operational risks, and to our best knowledge implemented for the first time in a business case, is to establish a relation between the random variables and decision variables. Demand is set to be dependent on decisions. The model becomes then far more complicated (nonlinear). In the standard form of stochastic programming, the decision variables and uncertainties are independent, see $[4,9,20,28]$. The other category of stochastic programs is the model with so called endogenous uncertainty, in which decision variables can influence the uncertainties, first addressed by Pflug [27]. While most work in non-standard problems is about the decision-dependent probability distributions, in this paper we are dealing with other uncertain elements in the model (e.g. demand) depending on decisions. The reader interested in further discussions of non-standard problems is referred to $[8,16,19]$ and the references therein. It is worth mentioning that our approach is in line with the risk management requirements defined by COSO II (Committee of Sponsoring Organization, www.coso.org) in the sense that the quality of Internal Control System affects the residual risks.

Another important aspect of the management of back offices is the notion of explicit and tacit knowledge (expertise), as explained above. In standardized operations, procedures enable the employees to solve most known problems. On the other hand, when a problem occurs which is not part of explicit (codified) knowledge, only the tacit knowledge (i.e. the expertise) can help to solve it. In our model we will thus assume that if more capacity of qualified workers is available this should lead to better service because mistakes in the 
operations are reduced; moreover this builds up the reputation and as a consequence the demand for such service increases. For this reason, the assumption of variable and parameter independence needs to be relaxed in our stochastic programming problems. However, this improvement leads to complicated models that are often non-convex.

Finally, for each developed model we use dual solutions to identify in the plan which scenarios are under stress regarding the availability of qualified workers. This analysis complements well the one provided by the primal solutions. Indeed it represents a way to price the risks due to lack of qualified resources. We focus on the main inventory constraints of the aggregate planning model, which are equality constraints with variables on both sides of the equations. In consequence, the dual solutions do not correspond to marginal values of resources. Nevertheless, dual variables (Lagrange multipliers associated with the constraints) give a relevant indication of the constraints that would need to be relaxed. For instance, they can show when liquidity should be available in supplement to be able to hire additional qualified workforce capacity.

The paper is organized as follows. In Section 2 we discuss the construction of the aggregate planning model adapted to our case study. In Section 3, we transform this model into a multistage stochastic programming problem first with random demand functions and second with random capacities. In Section 4, an approach is applied to revise probability distributions with Bayesian networks. In Section 5, we relax the usual assumption of stochastic programming that establishes an independence between decision variables and random variables. The results of the model's application are discussed in Section 6. In Section 7, we show how to employ dual solutions to identify situations that are at risk in the workforce plan, followed with conclusions and further research directions in Section 8.

\section{Presentation of Aggregate Planning Model}

Suppose we know with certainty all the parameters that are essential to make our planning decisions. The main task is to complete the transactions that are required in the back office of a bank. There are mainly two categories of professionals employed: qualified people and non-qualified people. Meanwhile temporary employees, as the recourse in this problem, are supposed to be available at any time, as needed. Our model must indicate how many of each group of people should be employed to satisfy the demand for transactions.

The qualified employees master both explicit knowledge as well as tacit knowledge (i.e. expertise). On the other hand the work of non-qualified employees essentially relies on explicit knowledge (i.e. written procedures). Consequently, the latter category of workers does not 
possess the sufficient skills to take the initiative when some tasks require more sophisticated knowledge or know-how. Accordingly, the two groups share different work efficiency and wages so that the numbers and proportion of each category of people employed will certainly influence the revenue of the company and may even further affect its sustainability, which will be discussed in subsequent sections.

The objective in our model is to minimize the costs and losses while maximizing the profit. The cost function includes three elements, i.e., hiring cost, firing cost and salaries paid to employees. Qualified people have higher firing cost and salaries than non-qualified people while hiring cost are assumed to be the same for both groups. We pay only salaries to temporary people without hiring or firing costs.

Profit is related with the volume of transactions successfully completed and hence defined as multiplication of this volume. As we impose satisfaction of all demands on time and there is no inventory of transactions, this volume is actually equivalent to the demand. Finally, profit is proportional to demand. It is worth mentioning that when demand does not change in the model, i.e., it is a parameter not a variable, profit is constant and does not need to be optimized in the objective. We have the following objective function:

$$
\min \sum_{t}\left(\left(x_{h}^{Q}\right)^{t}+\left(x_{h}^{N}\right)^{t}\right) h+\left(x_{f}^{Q}\right)^{t} f_{Q}+\left(x_{f}^{N}\right)^{t} f_{N}+\left(x^{Q}\right)^{t} w_{Q}+\left(x^{N}\right)^{t} w_{N}+\left(x^{P}\right)^{t} w_{P}
$$

where $t=1, \cdots, T$ is the time stage (a stage corresponds to a year); $x_{h}, x_{f}, x$ are the numbers of people hired, fired and kept. $-Q,-N$ and $-P$ stand for the quantities of Qualified people, Non-qualified people and temporary employees, and $h, f_{Q}, f_{N}, w_{Q}, w_{N}$ and $w_{P}$ stand for hiring cost, firing cost for qualified and non-qualified people, salaries per person for qualified, non-qualified and temporary employees, respectively. $x^{P}$ is the number of temporary personday, e.g. $x^{P}=1$ means a temporary employee working one day. In this model, we assume that both qualified and non-qualified people are kept at work at least a full year. Hence, $w_{Q}$ and $w_{N}$ are both yearly salaries. Temporary people are hired daily, which means $w_{P}$ is the payment to one temporary employee to work one day.

While we try to achieve the optimal value, there are two categories of constraints to be satisfied. The first group of constraints corresponds to the inventory of employees. Except for the first stage, we can hire and fire at each stage. Thus the number of employees we hold in a given period represents the number in previous stage subtracted by the number fired 
and increased by the number of newly hired. This is presented as follows:

$$
\begin{aligned}
\left(x^{Q}\right)^{t-1}+\left(x_{h}^{Q}\right)^{t}-\left(x_{f}^{Q}\right)^{t} & =\left(x^{Q}\right)^{t}, \quad \forall t \\
\left(x^{N}\right)^{t-1}+\left(x_{h}^{N}\right)^{t}-\left(x_{f}^{N}\right)^{t} & =\left(x^{N}\right)^{t}, \quad \forall t .
\end{aligned}
$$

The second one is the capacity constraint, which requires each demand to be completed on time at that stage. The capacities $\eta^{t}$ st stage $t$ are calculated in the following way:

$$
\left(x^{Q}\right)^{t} \alpha^{Q} \times 260+\left(x^{N}\right)^{t} \alpha^{N} \times 260+\left(x^{P}\right)^{t} \alpha^{P}=\eta^{t}, \quad \forall t
$$

where $\alpha^{Q}, \alpha^{N}$ and $\alpha^{P}$ are work capacities for qualified, non-qualified and temporary employees, respectively. These are numbers of transactions one person in the corresponding group can complete per day. We assume that $\alpha^{Q}>\alpha^{P}>\alpha^{N}$.

We suppose that there are 260 working days a year. The capacity represents the sum of transactions processed by qualified people, non-qualified people and temporary employees in a year. The capacity must be larger than or equal to the demand $\beta^{t}$.

To sum up, the full mathematical programming model can be written in the following way:

$$
\min \sum_{t}\left(\left(x_{h}^{Q}\right)^{t}+\left(x_{h}^{N}\right)^{t}\right) h+\left(x_{f}^{Q}\right)^{t} f_{Q}+\left(x_{f}^{N}\right)^{t} f_{N}+\left(x^{Q}\right)^{t} w_{Q}+\left(x^{N}\right)^{t} w_{N}+\left(x^{P}\right)^{t} w_{P}
$$

subject to

$$
\begin{gathered}
\left(x^{Q}\right)^{t-1}+\left(x_{h}^{Q}\right)^{t}-\left(x_{f}^{Q}\right)^{t}=\left(x^{Q}\right)^{t}, \quad \forall t=2, \cdots, T \\
\left(x^{N}\right)^{t-1}+\left(x_{h}^{N}\right)^{t}-\left(x_{f}^{N}\right)^{t}=\left(x^{N}\right)^{t}, \quad \forall t=2, \cdots, T \\
\left(x^{Q}\right)^{t} \alpha^{Q} \times 260+\left(x^{N}\right)^{t} \alpha^{N} \times 260+\left(x^{P}\right)^{t} \alpha^{P}=\eta^{t}, \quad \forall t=1, \cdots, T \\
\eta^{t} \geq \beta^{t}, \quad \forall t=1, \cdots, T \\
\left(x_{h}^{Q}\right)^{t},\left(x_{h}^{N}\right)^{t},\left(x_{f}^{Q}\right)^{t},\left(x_{f}^{N}\right)^{t},\left(x^{Q}\right)^{t},\left(x^{N}\right)^{t},\left(x^{P}\right)^{t} \geq 0 .
\end{gathered}
$$

where $t=1, \cdots, T$ is the planning horizon. The model above presents the standard form of Aggregate Planning Model, with (4a) as the cost objective function to be minimized, (4b) corresponding to the dynamic constraints and (4c), (4d) being the local constraints.

Figure 1 reflects a 5-period instance $(\mathrm{T}=5)$ [13]. Decision variables are the number of people to be employed. Consequently they are defined as integers, which makes that the model is an integer programming problem. However, in reality, it is not always true that we 


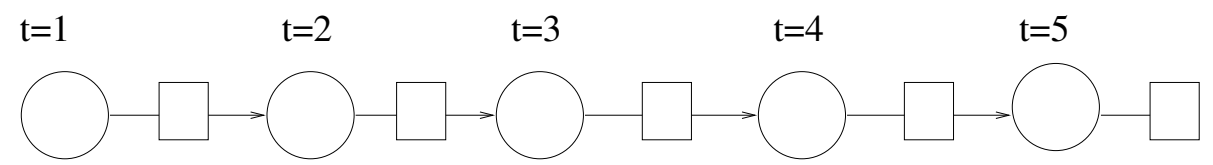

Figure 1: A deterministic multistage model representation: circles stand for chance nodes and rectangles stand for decisions.

know everything before making decisions. We will develop the stochastic aggregate planning model in the following section.

\section{Multistage Stochastic Programming}

In this section, we develop the stochastic programs with randomness in demand and capacities.

\subsection{Random Demand Parameters}

We can assume that the demands are random parameters and can thus take several possible values. This is usually modeled as an event tree (see for instance Figure 2). As a result, we obtain a multistage stochastic program.

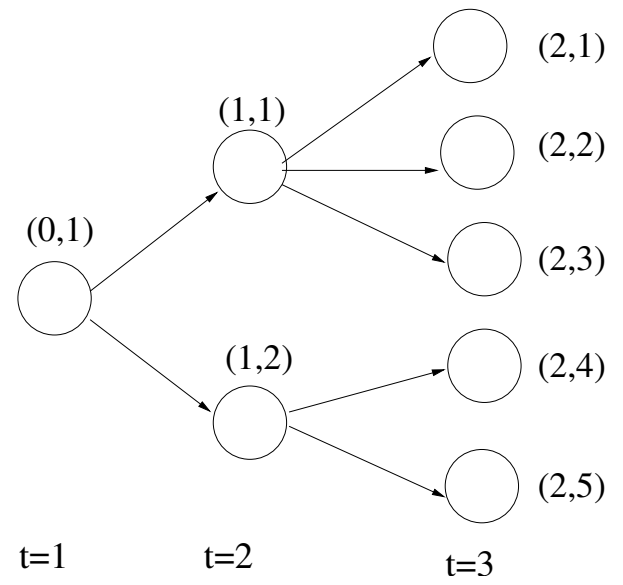

Figure 2: An example of event tree describing different demand states of nature.

The deterministic equivalent formulation of our aggregate planning model with uncertain demands becomes:

$\min \sum_{t, j} P_{j}^{t}\left(\left(\left(x_{h}^{Q}\right)_{j}^{t}+\left(x_{h}^{N}\right)_{j}^{t}\right) h+\left(x_{f}^{Q}\right)_{j}^{t} f_{Q}+\left(x_{f}^{N}\right)_{j}^{t} f_{N}+\left(x^{Q}\right)_{j}^{t} w_{Q}+\left(x^{N}\right)_{j}^{t} w_{N}+\left(x^{P}\right)_{j}^{t} w_{P}\right)$ 
subject to

$$
\begin{array}{r}
\left(x^{Q}\right)_{\pi_{j}}^{t-1}+\left(x_{h}^{Q}\right)_{j}^{t}-\left(x_{f}^{Q}\right)_{j}^{t}=\left(x^{Q}\right)_{j}^{t}, \quad \forall j, \quad \forall t=2, \cdots, T \\
\left(x^{N}\right)_{\pi_{j}}^{t-1}+\left(x_{h}^{N}\right)_{j}^{t}-\left(x_{f}^{N}\right)_{j}^{t}=\left(x^{N}\right)_{j}^{t}, \quad \forall j, \quad \forall t=2, \cdots, T \\
\left(x^{Q}\right)_{j}^{t} \alpha^{Q} \times 260+\left(x^{N}\right)_{j}^{t} \alpha^{N} \times 260+\left(x^{P}\right)_{j}^{t} \alpha^{P}=\eta_{j}^{t}, \quad \forall j, \quad \forall t=1, \cdots, T \\
\eta_{j}^{t} \geq \beta_{j}^{t}, \quad \forall j, \quad \forall t=1, \cdots, T
\end{array}
$$

where $j \in\{1, \cdots, J\}$, the set of demand state and $P$ is the probability distribution of demand, which defines (partial) path probabilities: $P_{j}^{t}$ is the probability (at the start) that a path goes through node $j$ at time $t$ and $\pi_{j}$ denotes the ancestor of node $j$ in the event tree. This formulation remains an integer programming problem.

\subsection{Random Capacity Parameters}

Due to some unpredictable events, like illness, holiday, or some unexpected accidents, constant capacity of employees cannot be guaranteed. Hence, we introduce uncertain capacity parameters into the model, $\widetilde{\alpha}^{Q}$ and $\widetilde{\alpha}^{N}$. Temporary employees are just employed in cases where we need more people. Consequently, we assume that they only take a small part of the total demand and thus we neglect the variation of their capacities. The corresponding capacity constraints become:

$$
\begin{array}{r}
\left(x^{Q}\right)_{j, l}^{t} \alpha_{l}^{Q} \times 260+\left(x^{N}\right)_{j, l}^{t} \alpha_{l}^{N} \times 260+\left(x^{P}\right)_{j, l}^{t} \alpha^{P}=\eta_{j, l}^{t}, \\
\eta_{j, l}^{t} \geq \beta_{j}^{t},
\end{array}
$$

where $l \in\{1, \cdots, L\}$, the set of all possible capacity values, $t=1, \cdots, T, j=1, \cdots, J$.

The other concern about capacity is new employees' capability. There is always a certain period for a person who is newly employed to get used to the work environment and become familiar with their responsibility. We cannot expect a new employee to be as efficient as an experienced one. Hence, their capabilities have to be valued separately. This is reflected in the following capacity constraint:

$$
\begin{aligned}
\left(\left(x^{Q}\right)_{j, l, v}^{t}-\left(1-\delta_{v}\right)\left(x_{h}^{Q}\right)_{j, l, v}^{t}\right) \alpha_{l}^{Q} \times 260 & +\left(\left(x^{N}\right)_{j, l, v}^{t}-\left(1-\delta_{v}\right)\left(x_{h}^{N}\right)_{j, l, v}^{t}\right) \alpha_{l}^{N} \times 260 \\
& +\left(x^{P}\right)_{j, l, v}^{t} \alpha^{P}=\eta_{j, l, v}^{t},
\end{aligned}
$$

where $t=1, \cdots, T, j=1, \cdots, J, l=1, \cdots, L$, and $v \in\{1, \cdots, V\}$, the set of all possible 
initial capability values $\delta$, where $\delta$ is the ratio of a new employee's capability by an experienced employee's capability, i.e., when an experienced employee completes one transaction, the new employee can do only $\delta$ transaction, where $0<\delta<1$. For a new hired employee, we have to subtract the lack of capacity $\left(1-\delta_{v}\right) \times\left(x_{h}^{Q}\right)_{j, l}^{t}$ and $\left(1-\delta_{v}\right) \times\left(x_{h}^{N}\right)_{j, l}^{t}$ from the total capacity. After one year, the new employee can work as efficiently as other employees.

The randomness in demand and capacity in the present model are both external risks which are not controlled by the decisions in the model. In a later section, we will exploit the internal risk from the uncertain demand, which depends on the decisions.

\section{Revising Operation Efficiency Probability Distribu- tions}

Operation efficiency is a way to measure the work done by employees, which is how many transactions an employee completes per unit time (labour cost) and how many errors an employee makes per transaction (error rate). In banking, a mistake in operations could bring big losses to the company and also decrease the demand in subsequent stages. Limiting the number of mistakes to the strict minimum is essential. This notion of operational efficiency is thus intimately linked to operational risk management $[14,6]$. It is related to people's knowledge and skills. Even if we know whether people are qualified or not, errors can still happen unexpectedly. Hence it is necessary to consider randomness of operation efficiency in the model. And we need the probability distribution describing the behavior of the random variable. However, in operational risk management, this kind of data is hard to collect. Firstly, long-term data is lacking, which means only the data of a few recent years is available. Secondly, a company never publishes its errors and operational losses and this makes the data unavailable. It is indeed extremely difficult to collect sufficient years of statistics describing operational risks to be then able to assume any theoretical behaviour. In such a case, we rely on Bayesian Network.

Bayesian Networks (BN) have emerged as a method of choice to deal with operational risks, especially in the banking sector, because their use does not necessitate the gathering of huge amounts of past data. BN is in fact grounded on classical decision theory and also adopts computing schemes of Artificial Intelligence [18]. Typically with a Bayesian approach we start with a subjective probability associated with a particular event. The a priori probability (also called subjective probability as opposed to objective probability) is assessed by the manager and corresponds to their own intuition and expertise. Along the way, obtaining new 
imperfect information and depending on the quality of past imperfect information provided by the issuer, the manager will be more or less inclined to modify his initial judgement. In a formal model this would be called the a posteriori analysis where a priori probabilities are modified using the Bayes formula. The Bayesian Network can be presented as a quantitative approach to handle qualitative dynamic choices. On the other hand, through the modeling of decision trees this approach should enable the manager to structure the dynamic dimension of the decision process.

Bayesian Network (BN) as a successful description of causalities has been widely applied in several areas like diagnosis, heuristic search, ecology, data mining and intelligent trouble shooting systems. It is defined as follows [18]:

Definition A Bayesian network consists of the following:

- A set of variables and a set of directed edges between states.

- Each variable has a finite set of mutually exclusive states.

- The variables together with the directed edges form a Directed Acyclic Graph(DAG). (A directed graph is acyclic if there is no directed path $A_{1} \rightarrow \cdots \rightarrow A_{n} \quad$ s.t. $A_{1}=A_{n}$.)

- To each variable $A$ with parents $B_{1}, \ldots, B_{n}$, there is attached the potential table $P\left(A \mid B_{1}, \ldots, B_{n}\right) . A$ is a child of $B$ and $B$ is a parent of $A$, if there is a link directed from $B$ to $A$. And, always, the parent(s) are set to be cause(s) of child.

$P\left(A \mid B_{1}, \ldots, B_{n}\right)$ is the conditional probability of $A$ given $\left(B_{1}, \ldots, B_{n}\right)$. Causal relations also have a quantitative side, namely their strength. This is expressed by attaching numbers to the links. In BN, it is natural to set the conditional probability to be a strength of the link. Let $B$ be a parent of $A, P(A \mid B)$ is the strength of their link.

The two fundamental rules for probability calculus are

$$
\begin{aligned}
P(A \mid B) P(B) & =P(A, B) \\
P(A \mid B) & =\frac{P(B \mid A) P(A)}{P(B)},
\end{aligned}
$$

where $P(A, B)$ is the probability of the joint event that both $A$ and $B$ happen. Sometimes $P(A \mid B)$ is called the likelihood of $B$ given $A$. Assume $A$ has $n$ outcomes $a_{1} \ldots a_{n}$, with an effect on the event $\mathrm{B}$, and we know B. Then, $P\left(a_{i} \mid B\right)$ is a measure of how likely it is that $a_{i}$ is the cause. In particular, if all $a_{i}$ 's have prior probabilities, Bayes' rule yields

$$
P\left(a_{i} \mid B\right)=\frac{P\left(B \mid a_{i}\right) P\left(a_{i}\right)}{P(B)} .
$$


In operational risk, unexpected variability in operation efficiency is the cause and has effect on losses. Applying BN in this context means using the information of loss we have, to revise the probability distribution of operation efficiency. We illustrate the use of BN in an example below.

Let $E r^{Q}$ be the operation efficiency of qualified people which is the number of errors made by qualified people in one transaction and similarly, let $E r^{N}$ be the operation efficiency of non-qualified employees. One error can definitely lead to a failure of the current transaction. We assume that $0 \leq E r^{Q} \leq E r^{N} \leq 1$. Firstly we need to know an a priori probability of the operation efficiency from expert's knowledge. For example, we denote $A$ as $E r^{Q} \geq \phi$ which means qualified employees have shown average poor efficiency and $\bar{A}$ as $\operatorname{Er}^{Q}<\phi$, where $\phi$ is a benchmark set to judge the operation efficiency. Suppose that the experts give the following estimates: $P(A)=0.2, P(\bar{A})=0.8$. In addition, denote $B$ as Loss $>0$ which is resulted by qualified people and $\bar{B}$ as Loss $=0$. Again, experts also determine for instance that $P(B \mid A)=0.7$ and $P(B \mid \bar{A})=0.1$. We can calculate

$$
P(B)=P(B \mid A) P(A)+P(B \mid \bar{A}) P(\bar{A})=0.22 .
$$

Now suppose that after one year, we know that loss happened in this year, which is the event B. We get new confidence about the operation efficiency by BN as

$$
P(A \mid B)=\frac{P(B \mid A) P(A)}{P(B)}=0.64
$$

In this simple example we notice that the probability of poor operation efficiency increases from 0.2 to 0.64 . For the random variables with more than 2 possible values, we still need to revise all the probabilities in the same way with Equation (13). In addition, after revising the probabilities of $A$, we also need to update $B^{\prime} s$ probabilities following the same way as Equation (12).

We can check that the revision process corresponds to our intuition. The conditional probability of positive loss given poor operation efficiency is higher than the unconditional probability when we know nothing about the operation efficiency, which is

$$
P(B \mid A) \geq P(B)
$$


Then, when loss happens, we revise $A^{\prime} s$ probability as follows:

$$
P(A \mid B)=\frac{P(A) P(B \mid A)}{P(B)} \geq P(A) .
$$

The inequality (15) follows easily from (14). This revision tells us that incurring loss increases the probability of poor operation efficiency, which agrees with people's intuitive judgement.

Finally, integrating the BN framework to revise the probability distribution of operation efficiency, denoted as $P_{-} O E$, in the aggregate planning model, we obtain the following equations:

$$
\begin{aligned}
\min & \sum_{t, j, l, v, s} P_{j, l, v}^{t} P_{\_} O E_{s}^{t}\left(\left(\left(x_{h}^{Q}\right)_{j, l, v, s}^{t}+\left(x_{h}^{N}\right)_{j, l, v, s}^{t}\right) h+\left(x_{f}^{Q}\right)_{j, l, v, s}^{t} f_{Q}+\left(x_{f}^{N}\right)_{j, l, v, s}^{t} f_{N}\right. \\
& +\left(x^{Q}\right)_{j, l, v, s}^{t} w_{Q}+\left(x^{N}\right)_{j, l, v, s}^{t} w_{N}+\left(x^{P}\right)_{j, l, v, s}^{t} w_{P} \\
& \left.+\left(\left(x^{Q}\right)_{j, l, v, s}^{t} \alpha_{l}^{Q} E r_{s}^{Q}+\left(x^{N}\right)_{j, l, v, s}^{t} \alpha_{l}^{N} E r_{s}^{N}\right) \times 260 \times \theta\right)
\end{aligned}
$$

subject to

$$
\begin{aligned}
& \left(x^{Q}\right)_{\pi_{(j, l, v, s)}^{t-1}}+\left(x_{h}^{Q}\right)_{j, l, v, s}-\left(x_{f}^{Q}\right)_{j, l, v, s}^{t}=\left(x^{Q}\right)_{j, l, v, s}^{t} \quad, \\
& \left(x^{N}\right)_{\pi_{(j, l, v, s)}^{t-1}}+\left(x_{h}^{N}\right)_{j, l, v, s}^{t}-\left(x_{f}^{N}\right)_{j, l, v, s}^{t}=\left(x^{N}\right)_{j, l, v, s}^{t} \\
& \forall j, \quad \forall l, \quad \forall v, \quad \forall s, \quad \forall t=2, \cdots, T, \\
& \left(\left(x^{Q}\right)_{j, l, v, s}^{t}-\left(1-\delta_{v}\right)\left(x_{h}^{Q}\right)_{j, l, v}^{t}\right) \alpha_{l}^{Q} \times 260+\left(\left(x^{N}\right)_{j, l, v, s}^{t}-\left(1-\delta_{v}\right)\left(x_{h}^{N}\right)_{j, l, v}^{t}\right) \alpha_{l}^{N} \times 260 \\
& +\left(x^{P}\right)_{j, l, v, s}^{t} \alpha^{P}=\eta_{j, l, v, s}^{t} \\
& \eta_{j, l, v, s}^{t} \geq \beta_{j, l, v, s}^{t}, \quad \forall j, \quad \forall l, \quad \forall v, \quad \forall s, \quad \forall t, \\
& P \_O E_{s}^{t}=\frac{P_{-} O E_{\pi_{s}}^{t-1} P_{\text {loss }}(\text { Loss }>0 \mid s)}{P_{\text {loss }}^{t-1}(\text { Loss }>0)}, \quad \forall s, \quad \forall t=2, \cdots, T, \\
& \text { if loss happens, } \\
& P_{-} O E_{s}^{t}=\frac{P_{-} O E_{\pi_{s}}^{t-1}\left(1-P_{\text {loss }}(\text { Loss }>0 \mid s)\right)}{1-P_{\text {loss }}^{t-1}(\text { Loss }>0)}, \quad \forall s, \quad \forall t=2, \cdots, T, \\
& \text { if no loss happens, } \\
& P_{\text {loss }}^{t}(\text { Loss }>0)=\sum_{s} P_{\text {loss }}^{t-1}(\text { Loss }>0 \mid s) P_{-} O E(s), \quad \forall s, \quad \forall t=2, \cdots, T,
\end{aligned}
$$

where $j=1, \cdots, J, l=1, \cdots, L, v=1, \cdots, V$, and $s \in\{1, \cdots, S\}$, the set of operation efficiency and $\pi_{s}$ is the parent node of $s . \theta$ is the loss in capital per error. Equations (16e) impose the Bayesian Network, where $P_{-} O E$ is the probability distribution of operation efficiency, $P_{\text {loss }}$ is the probability distribution of the loss while $P_{\text {loss }}(\mid)$ is the conditional probability 
distribution of loss conditioned on the operation efficiency. Equations (16ea) and (16eb) are the probability revisions of operation efficiency. At each stage $t$, we revise the probabilities of previous stage $t-1$ by collecting the loss information. The probability distribution revised at each stage is essential in the objective function and clearly influences the decision-making accordingly. Equation (16ec) calculates the new probability of loss after operation efficiency probability revision at each stage. As time progresses, we continuously collect information to update the probability distributions, see Figure 3. The optimization problem (16) is an linear integer program.

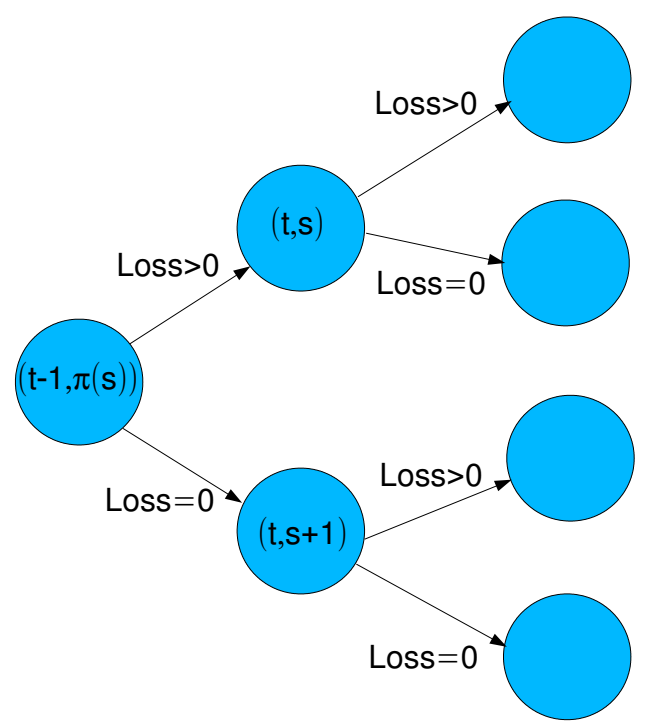

Figure 3: The process of probability distribution revision, where $\pi(s)$ is the ancestor node of $s$.

\section{Random Parameters Dependent on Decisions}

Demand can be dependent upon several factors, like the trend of the market or the management of the company. One of the most important factors is the reputation acquired based on the quality of transactions processed so far, which is essentially related to the individual employee expertise. When customers receive products or services of high quality, they are more likely to continue the business and even increase the volume or bring more business to the company, which will increase the demand at next stage. Conversely, if customers are not satisfied with what they have got, they may change to other companies.

The operational risk in this case has two issues. Firstly, demand may decrease due to the low quality of service provided and this results in revenue drop. According to COSO philosophy, the crude risk is reduced in function of the level of quality of the internal control 
system. As an example, we can imagine that a bank's main activity is in the development and trading of structured products (the crude risk is thus huge in terms of financial and operational risks). However, if highly qualified people are dealing with these activities, the internal control system presents a high level of quality. Consequently, the residual risk is minimized. The managerial treatment of risk becomes thus crucial in regard of the COSO philosophy. Another element that has to be taken into account in the management of back offices is the notion of explicit and tacit knowledge arising from service science. Typically in a back office, tasks are very standardized and documented in working procedures. These tasks correspond to the explicit knowledge. Tacit knowledge on the other hand as defined by [22] corresponds to information that is difficult to express, formalize or share, in contrast to explicit knowledge, which is conscious and can be put into words. When something unexpected happens that might affect the service production, which is not included in the procedures, solely the expertise of qualified workers will permit correction of the problem and a return to normal. This statement is confirmed for instance in [23] that qualitative skills, like information search style, level of education and training on risk, influence the capability of risk manager to identify risks. Hence, we assume that if more capacity of qualified workers is available, this should lead to better service.

Our modified aggregate planning model will indicate how many of the qualified and nonqualified people should be employed. Here comes the second issue of the risk. If demand increases because of excellent services received by customers and good reputations built up, the lack of workforce is at risk. Such demand growth is largely determined by the decisions of the model, i.e. volume of qualified people, and cannot be captured by statistic behavior prediction. It is thus essential to take into account the relations of demand and decision in the model in order to properly manage the risk of lack of expertise. This also illustrates the problem that operational risk in this perspective cannot be treated in the same way as market risk. Indeed, market risks result from market fluctuations which are by definition non-controllable. This is not the case for operational risks whose origins are human and can, to a certain extent, often be controlled internally.

Operation efficiency is used to measure the work done by employees. Qualified people have additional professional knowledge and skills enabling them to achieve a higher throughput with a lower rate of error than their non-qualified colleagues. However, on the other hand, non-qualified employees are much cheaper to employ in both salary and firing terms. We attempt to determine the number of qualified people required to minimize losses due to employee error, hence not only directly impacting profits, but also growing demand in subsequent stages due to a better customer experience. We are therefore trading off the additional 
cost of qualified employees against the reduction in error-based losses and the growth in demand they produce.

To measure employees' work in a macro view, we use the total number of errors that employees make in a year, denoted as $\sharp E$ :

$$
\sharp E=\left(\left(x^{Q}\right) \alpha^{Q} E r^{Q}+\left(x^{N}\right) \alpha^{N} E r^{N}\right) \times 260 .
$$

As the reputation could be ruined by errors, one error will lead to a miss of more than one unit of demand. The decrease of demand due to errors can be:

$$
\beta_{\text {dec }}=(\sharp E)^{\tau},
$$

where $1 \leq \tau \leq 2$. Meanwhile, the transactions done correctly, denoted as $\sharp C$ can bring more business, which means the demand can increase by the following amount:

$$
\beta_{\text {inc }}=\lambda \sharp C,
$$

where $0<\lambda<1$.

When operation efficiency is not considered, demand can be estimated according to the market and other conditions, denoted as $\beta^{\prime}$. Due to operation efficiency, the value of demand could differ from $\beta^{\prime}$ in the following way:

$$
\beta=\beta^{\prime}+\beta_{i n c}-\beta_{\text {dec }}=\beta^{\prime}+\lambda \sharp C-\sharp E^{\tau},
$$

where $0<\lambda<1,1 \leq \tau \leq 2$. Nonlinearity is introduced by this function. This polynomial function, however, can be linearized by Special Order Set 2 (SOS2), see [30].

Now, demand is changing with decisions, which makes the profit dependent on decisions too and therefore we take it into account in the objective function: it contributes a term $\beta^{t} \cdot \gamma$, where $\gamma$ is the income per transaction completed. Hence, the model becomes:

$$
\begin{aligned}
\min & \sum_{t, j, l, v, s} P_{j, l, v}^{t} P_{-} O E_{s}^{t}\left(\left(\left(x_{h}^{Q}\right)_{j, l, v, s}^{t}+\left(x_{h}^{N}\right)_{j, l, v, s}^{t}\right) h+\left(x_{f}^{Q}\right)_{j, l, v, s}^{t} f_{Q}+\left(x_{f}^{N}\right)_{j, l, v, s}^{t} f_{N}\right. \\
& +\left(x^{Q}\right)_{j, l, v, s}^{t} w_{Q}+\left(x^{N}\right)_{j, l, v, s}^{t} w_{N}+\left(x^{P}\right)_{j, l, v, s}^{t} w_{P} \\
& \left.+\left(\left(x^{Q}\right)_{j, l, v, s}^{t} \alpha_{l}^{Q} E r_{s}^{Q}+\left(x^{N}\right)_{j, l, v, s}^{t} \alpha_{l}^{N} E r_{s}^{N}\right) \times 260 \times \theta-\beta_{j, l, v, s}^{t} \gamma\right)
\end{aligned}
$$


subject to

$$
\begin{aligned}
& \left(x^{Q}\right)_{\pi_{(j, l, v, s)}^{t-1}}+\left(x_{h}^{Q}\right)_{j, l, v, s}-\left(x_{f}^{Q}\right)_{j, l, v, s}^{t}=\left(x^{Q}\right)_{j, l, v, s}^{t} \quad, \\
& \left(x^{N}\right)_{\pi_{(j, l, v, s)}}^{t-1}+\left(x_{h}^{N}\right)_{j, l, v, s}^{t}-\left(x_{f}^{N}\right)_{j, l, v, s}^{t}=\left(x^{N}\right)_{j, l, v, s}^{t}, \\
& \forall j, \quad \forall l, \quad \forall v, \quad \forall s, \quad \forall t=2, \cdots, T, \\
& \left(\left(x^{Q}\right)_{j, l, v, s}^{t}-\left(1-\delta_{v}\right)\left(x_{h}^{Q}\right)_{j, l, v, s}^{t}\right) \alpha_{l}^{Q} \times 260+\left(\left(x^{N}\right)_{j, l, v, s}^{t}-\left(1-\delta_{v}\right)\left(x_{h}^{N}\right)_{j, l, v, s}^{t}\right) \alpha_{l}^{N} \times 260 \\
& +\left(x^{P}\right)_{j, l, v, s}^{t} \alpha^{P}=\eta_{j, l, v, s}^{t}, \\
& \eta_{j, l, v, s}^{t} \geq \beta_{j, l, v, s}^{t} \quad, \quad \forall j, \quad \forall l, \quad \forall v, \quad \forall s, \quad \forall t, \\
& \sharp E_{j, l, v, s}^{t}=\left(\left(x^{Q}\right)_{j, l, v, s}^{t} \alpha_{l}^{Q} \operatorname{Er}_{s}^{Q}+\left(x^{N}\right)_{j, l, v, s}^{t} \alpha_{l}^{N} \operatorname{Er}_{s}^{N}\right) \times 260, \quad \forall j, \quad \forall l, \quad \forall v, \quad \forall s, \quad \forall t, \text { (20ea) } \\
& \sharp C_{j, l, v, s}^{t}=\beta_{j, l, v, s}^{t}-\sharp E_{j, l, v, s}^{t} \quad, \quad \forall j, \quad \forall l, \quad \forall v, \quad \forall s, \quad \forall t, \\
& \beta_{j, l, v, s}^{t+1}=\beta_{j, l, v, s}^{\prime t+1}+\lambda \sharp C_{\pi_{(j, l, v, s)}^{t}}^{t}-\left(\sharp E_{\pi_{(j, l, v, s)}^{t}}^{t}\right)^{\tau}, \quad \forall j, \quad \forall l, \quad \forall v, \quad \forall s, \quad \forall t, \\
& P_{-} O E_{s}^{t}=\frac{P_{-} O E_{\pi_{s}}^{t-1} \times P_{\text {loss }}(\text { Loss }>0 \mid s)}{P_{\text {loss }}^{t-1}(\text { Loss }>0)}, \quad \forall s, \quad \forall t=2, \cdots, T, \\
& \text { if loss happens, } \\
& P_{-} O E_{s}^{t}=\frac{P_{-} O E_{\pi_{s}}^{t-1} \times\left(1-P_{\text {loss }}(\text { Loss }>0 \mid s)\right)}{1-P_{\text {loss }}^{t-1}(\text { Loss }>0)}, \quad \forall s, \quad \forall t=2, \cdots, T, \\
& \text { if no loss happens, } \\
& P_{\text {loss }}^{t}(\text { Loss }>0)=\sum_{s} P_{\text {loss }}^{t-1}(\text { Loss }>0 \mid s) \times P_{-} O E(s), \quad \forall s, \quad \forall t=2, \cdots, T,
\end{aligned}
$$

where $j=1, \cdots, J, l=1, \cdots, L, v=1, \cdots, V, s=1, \cdots, S$. Since the nonlinear demand function $(20 \mathrm{ec})$ can be linearized, this model still can be easily solved by integer solvers.

It is worth mentioning that while demand is influenced by previous decision, there exists also an influence in the opposite direction. $\beta^{t}$ is one of the main factors changing decision at stage $t$. Conversely decision at stage $t$ affects those at stage $t-1$. Hence the influence of $\beta^{t}$ on $t$-stage-decisions is spread to $t-1$-stage-decisions, which is shown in Figure 4.

\section{Implementations of the Models}

All models discussed in this paper are written in AMPL and solved by CPLEX, including the linearized form of model (20). Consider a 3-stage-problem, suppose each random variable has 2 possible values and there are 4 random variable sets, i.e., demand, capacities, initial 


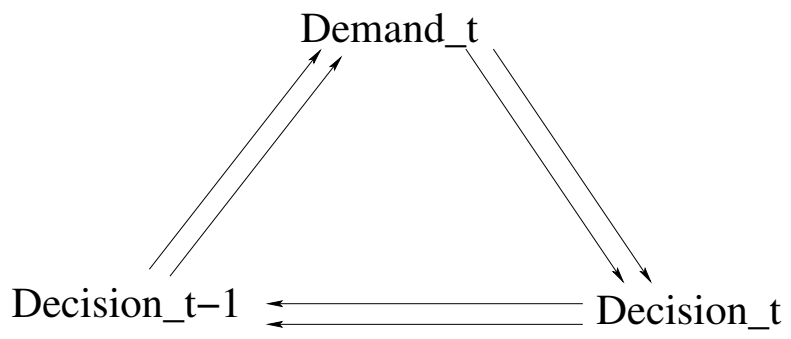

Figure 4: Influence Chart

capability and operation efficiency. Hence, there are 4369 nodes in total for this 3 -stageproblem. At each node there are 7 integer decision variables and one continuous demand variable. Overall, there are 30583 decision variables in the model. In addition, 2 inventory constraints, one capacity constraint and 1 demand constraint at each node sum up to 17476 constraints.

This aggregated planning model is trying to help decision makers to get the optimal decisions while satisfying the demand and controlling the risk. Our effort is focused on risk management. As discussed above, qualified people armed with better knowledge and skills are considered to be safer to the company than non-qualified people. We test four different models, i.e., basic model, model with random capacity, model with $\mathrm{BN}$ revision and model with demand function dependent on decisions. The basic model is referred to the stochastic programming model assuming the randomness only in demand and no dependence between decisions and random variables, as given by Equations (5) in Section 3. The model with random capacity corresponds to Equations (6) - (8), BN revision is governed by Equation (16e). Demand function depends on decisions through Equations (20e). The solution of the basic model excluding risk factors suggests employing more non-qualified people than the more elaborate models to go for highest profit. The decisions provided by the basic model are questionable since they expose the company to the risk of demand satisfying failure and operation errors. In the other models, we can see an average increase of employment of qualified people. Tables 1, 2 and 3 show the summary of results of each model. We report in them the numbers of qualified and non-qualified people to be employed as determined by an appropriate optimization model. We solve a 3 -stage problem but we are really concerned with the decisions at first stage and only these numbers are reported in the tables. In results presented in Tables 1 and 2, the risk is from random demand, random capacities and probability distributions, which are all principally resulting from market risks and consequently cannot be controlled. The analysis of results collected in Table 3, by considering the dependence between the decisions and variables, suggests that the controllable risks require 
more qualified people. By taking into account the operational risk in random capacity and the demand depending on decisions and constructing more reliable probability distribution, the model makes the decision to employ more qualified people than the basic model, so that the operation is more secure. Meanwhile, the cost, profit and loss are well balanced. In addition, the model with demand function depending on decisions reflects an average increase of demand of $5.48 \%$.

\begin{tabular}{|c|c|c|}
\hline Models & Basic Model & Random Capacity \\
\hline Qualified People & 2 & 4 \\
\hline Non-Qualified People & 51 & 43 \\
\hline
\end{tabular}

Table 1: Comparison between the basic model and the model with random capacity.

\begin{tabular}{|c|c|c|}
\hline Models & Basic Model & BN Revision \\
\hline Qualified People & 2 & 6 \\
\hline Non-Qualified People & 51 & 35 \\
\hline
\end{tabular}

Table 2: Comparison between the basic model and the model with BN revision.

\begin{tabular}{|c|c|c|}
\hline Models & Basic Model & Demand Function \\
\hline Qualified People & 2 & 9 \\
\hline Non-Qualified People & 51 & 23 \\
\hline
\end{tabular}

Table 3: Comparison between the basic model and the model with demand function depending on decisions.

It is natural that the number of employees decreases when the corresponding cost (e.g. hiring, firing cost or salaries) increases. On the other hand, if people improve their skills, which means they can deal with more transactions or make fewer errors than before, they are more valuable for their employers. In our case study parameter $\theta$ has more influence on decisions made than $\gamma$, i.e., the decrease of $\theta$ pushed down the number of qualified people employed. We have also looked at the evolution of employment over the planning period (3 stages) and we have observed that the number of non-qualified people does not change a lot. When demand varies from stage to stage, qualified people are more frequently fired or hired.

In the Appendix, we present some details of an approximated solution associated with the nonlinear model described by Equations (20).

\section{$7 \quad$ The Pricing of Operational Risk}

In the case of a convex nonlinear programming problem with inequality constraints, the dual prices correspond to the Lagrange multipliers. Their interpretation is similar to that of the 
shadow prices in linear programming. For an additional unit of the right hand side parameter of a given constraint the associated Lagrange multiplier indicates by how many units the objective function will vary, while other quantities remain the same.

In our context, the shadow price of the constraint describing the availability of qualified workers gives the value of an additional hour of expertise provided by a qualified employee. In terms of risk management, we obtain pricing information to set up a kind of "strategic reserve" (terms from the military science designing a supplementary force available and ready to act just in case of urgent needs).

Nowadays, in business this notion of strategic reserve for dealing with operational risk is not accepted. Generating a significant cost to hire expertise just to be able to solve difficult operations problems in case they would arise is not considered to be viable. However we believe that our model enables the risk budget planner to address the necessity to plan sufficient expertise in order to deal with unexpected operational problems. Moreover the dual solution represents a relevant way to quantify the expertise dedicated to risk management. The shadow price in the context of stochastic programming to produce a uniform $\mathrm{CO}_{2}$ tax was first applied in a result analysis by Bahn et al. in [3].

\subsection{The Shadow Price in Optimization}

The Lagrangian associated with the nonlinear programming problem:

$$
\min f(x)
$$

subject to

$$
\begin{aligned}
& g(x)<b, \\
& h(x)=0,
\end{aligned}
$$

has the following form

$$
L(x, y, z)=f(x)+y^{T}(g(x)-b)+z^{T} h(x),
$$

where $y \geq 0$ and $z$ are called Lagrange multipliers. We have

$$
\min _{x} \max _{y, z} L(x, y, z)=\min f(x) .
$$


Hence, instead of solving the original model, the optimal value can be obtained by the Lagrange function. This is the basic tool in solving nonlinear programs. We know that the optimal value of $L(x, y, z)$ must be achieved at the stationary point. By taking the first derivative of the Lagrangian, we obtain the first order optimality condition,

$$
\frac{d f(x)}{d x_{j}}+\sum y_{i} \frac{d g_{i}(x)}{d x_{j}}+\sum z_{k} \frac{d h_{k}(j)}{d x_{j}}=0 .
$$

The multiplier $y_{i}$ of inequality constraint can be (locally) interpreted as follows: one unit increase of resource $g_{i}$ will lead to $y_{i}$ unit decrease of objective function $f(x) . y_{i}$ are also defined as the shadow prices or dual prices. If $y_{i}$ is a relatively large value compared to others, to achieve the optimal objective value, the corresponding resource will be a lower priority to be increased than others. The Lagrange multiplier $z_{k}$ associated with the equality constraint indeed measures the "force" of this equality constraint. However, observe that in general Lagrange multipliers depend on the scaling of constraints. Hence, the same problem after scaling has a different Lagrange multiplier. In our case, all constraints have similar scaling and we can compare the associated Lagrange multipliers, at least we can use them to provide us with a qualitative insight.

We have developed a fourth category of model with some approximations to make it convex and smooth (though nonlinear). Keeping the continuity property enables us to produce shadow prices (Lagrange multipliers in the case of convex nonlinear models) which gives the implicit value of resources. In that case we obtain information related to value of expertise of qualified workers. To our knowledge this is the first time that shadow prices are used to value the expertise of workers in an operations risk management context.

\subsection{Exploiting the Shadow Pricing Approach}

In model (20), we have two groups of constraints: inventory constraints (20b) correspond to Equation (21c) and capacity constraints (20d) correspond to Equation (21b). Let us focus on the inventory constraints that express the balance between employees hired, fired and held at each node of both qualified and non-qualified people. Since Lagrange multipliers associated with equality constraints such as inventory equations (20b) depend on the scaling of these constraints, we need to be careful and keep their original scaling. Then we can compare the magnitude of the absolute values of Lagrange multipliers associated with these constraints and deduce from such a comparison which constraints are tight.

Tables 4, 5, 6 and 7 show the shadow prices of constraints in the four different models explained in Section 6. In each table, the absolute values of Lagrange multipliers associated 
with qualified people inventory constraints are in general greater than those of non-qualified people which means these constraints are tight. In terms of risk management we interpret this fact as a warning that more attention should be paid to the availability of qualified workers than that of non-qualified workers. For each node in every time period we identify the greatest danger to be a shortage of qualified people. In other words, employing more qualified people in the improved models, i.e. models with random capacity, demand function, etc, will decrease the operational risk. This can also be illustrated in the following shadow prices comparison tables. Tables 5, 6 and 7 show the shadow prices of the inventory constraints in the model with random capacity, model with $\mathrm{BN}$ and the model with demand function depending on decisions, respectively. There is a general decrease in all absolute values of Lagrange multipliers in these three models, while the risk also shrinks.

\begin{tabular}{|c|c|c|c|c|c|c|}
\hline Shadow Prices & Node 1 & Node 2 & Node 3 & Node 4 & Node 5 & Node 6 \\
\hline Inventory Constraint, Q People & 7500 & -2000 & -7500 & 3000 & -5000 & 2000 \\
\hline Inventory Constraint, NQ People & 570 & -1445 & -1800 & 1650 & -1075 & 1100 \\
\hline Capacity Constraints & 30.5769 & 27.1154 & 14.5385 & 15 & 9.9808 & 10 \\
\hline
\end{tabular}

Table 4: Shadow prices of inventory constraints and capacity constraints in basic model.

\begin{tabular}{|c|c|c|c|c|c|c|}
\hline Shadow Prices & Node 1 & Node 2 & Node 3 & Node 4 & Node 5 & Node 6 \\
\hline Qualified People & 1645 & 810 & -458 & 840 & -146 & 560 \\
\hline Non-Qualified People & -81 & -121 & -75 & 372 & 36 & 248 \\
\hline
\end{tabular}

Table 5: Shadow prices of inventory constraints in model with random capacity.

\begin{tabular}{|c|c|c|c|c|c|c|}
\hline Shadow Prices & Node 1 & Node 2 & Node 3 & Node 4 & Node 5 & Node 6 \\
\hline Qualified People & 2028 & -541 & 575 & -230 & 383 & -153 \\
\hline Non-Qualified People & 136 & -375 & 88 & -109 & 62 & -72 \\
\hline
\end{tabular}

Table 6: Shadow prices of inventory constraints in model with BN.

\begin{tabular}{|c|c|c|c|c|c|c|}
\hline Shadow Prices & Node 1 & Node 2 & Node 3 & Node 4 & Node 5 & Node 6 \\
\hline Qualified People & 7500 & -2000 & -3000 & 2000 & -3000 & 2000 \\
\hline Non-Qualified People & 430 & -1304 & -1650 & 1100 & -1650 & 1100 \\
\hline
\end{tabular}

Table 7: Shadow prices of inventory constraints in model with demand function depending on decisions.

\section{Conclusion}

Aggregate planning models as a category of mathematical programming models deal with the basic production or operation management problems. The multistage aggregate planning 
model mainly focuses on the labor allocation management problem. By satisfying the demand constraints at each stage, optimal staff allocation is determined while minimizing costs (including salaries, hiring and firing costs) and losses, resulting from erroneous operations.

In the context of real enterprise risk management, decisions must be made that will affect future choices and outcomes. Hence when considering future events in business activity planning, it is pertinent to take into account uncertain parameters within the planning model. This is often done using a multistage stochastic programming model.

Although stochastic programming is a planning tool that simultaneously takes into account cause-and-effect relations and random variables, most applications in financial risks have been limited to the case where random variables are assumed to follow some theoretical probability distribution functions. In order to add more relevance to the risk planning process of banking operations, we have combined the methodology of Bayesian networks with aggregate planning models.

In general, the demand (a parameter of the model) is assumed to be independent of decisions. However, in reality, this is often not the case. If we consider for example the reputation of companies - the demand could be dependent upon the success of previous decisions; when customers receive products or services of high quality, they are more likely to continue the business and even increase its volume, which will increase the demand at next stage. Conversely, if customers are not satisfied with the service provided, they may change to other companies. This problem has been addressed in our stochastic aggregate planning model by establishing a link between the random parameters and the decision variables. In particular, our model is in line with the COSO risk management philosophy assuming that the quality of the Internal Control Systems affects the residual risks. Simply said operations risks are controllable through good management and this is what we take into account in our model for the first time.

This latter model results in a mixed integer problem that we have solved with CPLEX. Finally, we interpret interesting results obtained with this methodology that confirms that our modeling concept is relevant. Additionally, shadow prices of inventory constraints are used to price the risks of operations. Our model indicates at which period money should be set aside to be able to hire sufficient qualified workforce if needed.

This model is intended to support decision-making processes regarding employment strategies in order to manage operational risk from a human perspective. After studying the work efficiency and other kinds of skills of existing and potential employees, the management can input these coefficients into the model along with demand predictions. Then the model will generate an optimal strategy involving the proper workforce categories reparti- 
tion minimizing the risk of inadequate expertise. It must be also added that the tractability of the model presented means that it can be implemented and solved by most commercial optimization codes.

To further deal with the work efficiency, we intend to apply learning curves to the model that describe the employees' learning process more precisely. In addition, the notion of service delay will also be considered in the model, which means we relax the assumption that we always have enough temporary employees as back-up.

\section{Acknowledgement}

The authors are grateful to the anonymous referees for their helpful remarks and to $\mathrm{Mr}$ Kristian Woodsend for a careful reading of the paper.

\section{References}

[1] What's next for India: Beyond the back office. The Boston Consulting Group, 2005. Special Report.

[2] W. J. Abernathy, N. Baloff, J. C. Hershey, and S. Wandel, A three-stage manpower planning and scheduling model-a service-sector example, Operations Research, 21 (1973), pp. 693-711.

[3] O. Bahn, E. Fragnière, And S. Kypreos, Swiss energy taxation options to curb $\mathrm{co}_{2}$ emissions, European Enviroment, (1998), pp. 94-101.

[4] J. R. Birge and F. Louveaux, Introduction to Stochastic Programming, SpringerVerlag, 1997.

[5] L. BRINK AND B. MCCARL, The tradeoff between expected return and risk among cornbelt farmers, American Journal of Agricultural Economics, 60 (1978), pp. 259-263.

[6] J. Debely, M. Dubosson, and E. Fragnière, The travel agent: Delivering more value by becoming an operational risk manager, in 9th International Research Seminar in Service Management, 2006, pp. 178-203.

[7] M. Dubosson, E. Fragnière, and B. Milliet, A control system designed to address the intangible nature of service risks, in IEEE International Conference on Service Operations and Logistics, and Informatics, 2006, pp. 90-95.

[8] J. DuPAC̆OvÁ, Optimization under exogenous and endogenous uncertainty, in MME06, L. Lukáš, ed., University of West Bohemia in Pilsen, 2006.

[9] J. DupaC̆OvÁ, J. HurT, And J. ŠtĚPÁn, Stochastic Modeling in Economics and Finance, Kluwer Academic Publishers, 2002. 
[10] B. P. Dzielinski And R. E. Gomory, Optimal programming of lot sized, inventory and labour allocations, Management Science, 11 (1965), pp. 874-890.

[11] R. J. EBERT, Aggregating planning with learning curve productivity, Management Science, 23 (1976), pp. 171-182.

[12] D. E.Kroll AND K. R. Kumar, The incorporation of learning in production planning models, Annals of Operations Research, 17 (1989), pp. 291-304.

[13] E. FragniÈre And J. GondZIO, Stochastic programming from modelling languages, in Applications of Stochastic Programming, S. W. Wallace and W. T. Ziemba, eds., SIAM Series on Optimization, 2002, pp. 1-21.

[14] E. Fragnière And G. Sullivan, Risk Management-Safeguarding Company Assets, Thomson, 2007.

[15] J. GierA, Bpo in banking: Outsourcing the back office. Forrester Research, Nov 2004.

[16] V. Goel and I. E. Grossmann, A class of stochastic programs with decision dependent uncertainty, Mathematical Programming, 108 (2006), pp. 355-394.

[17] S. C. Graves, A review of production scheduling, Operations Research, 29 (1981), pp. $646-675$.

[18] F. V. Jensen, Bayesian Networks and Decision Graphs, Springer, 2001.

[19] T. W. Jonsbråten, R. J.-B. Wets, And D. L. Woodruff, A class of stochastic programs with decision dependent random elements, Annals of Operations Research, 82 (1998), pp. 83-106.

[20] P. Kall and S. W. Wallace, Stochastic Programming, John Wiley \& Sons, 1994.

[21] L. J. KRajewski And H. E. Thompson, Efficient employment planning in public utilities, The Bell Journal of Economics, 6 (1975), pp. 314-326.

[22] R. LUBIT, Tacit knowledge and knowledge management: The keys to sustainable competitive advantage, Organizational Dynamics, 29 (2001), pp. 164-178.

[23] E. Maytorena, G. M. Winch, J. Freeman, and T. Kiely, The influence of experience and information search styles on project risk identification performance, IEEE Transactions on Engineering Management, 54 (2007), pp. 315-326.

[24] P. MCCONnell And K. BlaCker, An approach to modelling operational risk in banks.

[25] D. P. Morton And E. Popova, A Bayesian stochastic programming approach to an employee scheduling problem, IIE Transactions, 36 (2004), pp. 155-167. 
[26] A. Parasuraman, V. A. Aeithaml, and L. L. Berry, A conceptual model of service quality and its implications for future research, Journal of Marketing, 49 (1985), pp. 4150 .

[27] G. Pflug, Online optimization of simulated Markov processes, Mathematics of Operations Research, 15 (1990), pp. 381-395.

[28] A. PrÉKopA, Stochastic Programming, Kluwer Academic Publishers, 1995.

[29] G. H. SAAD, Hierarchical production-planning systems: Extensions and modifications, Journal of the Operational Research Society, 41 (1990), pp. 609-624.

[30] H. P. Williams, Model building in mathematical programming, Wiley, 1978.

[31] Y. YASUdA, Application of Bayesian Inference to Operational Risk Management, $\mathrm{PhD}$ thesis, University of Tsukuba, 2003.

\section{A Appendix}

This Appendix shows part of the solutions to the model given by Equations (20).

\begin{tabular}{|c|c|c|c|}
\hline Employees & Qualified & Non-Qualified & Temporary \\
\hline No. Held & 14 & 3 & 0 \\
\hline
\end{tabular}

Table 8: Decisions at first stage: numbers of people held.

\begin{tabular}{|c|c|c|c|c|c|c|c|}
\hline node $j$ & node $l$ & node $v$ & node $s$ & Qualified & Non-qualified & Temp & Demand \\
\hline 1 & 1 & 1 & 1 & 8 & 3 & 34 & 11474 \\
\hline 1 & 2 & 1 & 1 & 14 & 4 & 11 & 18474 \\
\hline 1 & 3 & 1 & 1 & 8 & 3 & 33 & 11474 \\
\hline 1 & 4 & 1 & 1 & 14 & 4 & 11 & 18474 \\
\hline 2 & 1 & 1 & 1 & 8 & 3 & 37 & 11483 \\
\hline 2 & 2 & 1 & 1 & 14 & 1 & 0 & 15575 \\
\hline 2 & 3 & 1 & 1 & 12 & 0 & 0 & 15574 \\
\hline 2 & 4 & 1 & 1 & 17 & 4 & 15 & 21574 \\
\hline 3 & 1 & 1 & 1 & 12 & 0 & 0 & 15575 \\
\hline 3 & 2 & 1 & 1 & 18 & 1 & 0 & 21575 \\
\hline 3 & 2 & 1 & 3 & 17 & 4 & 10 & 21561 \\
\hline
\end{tabular}

Table 9: Part results at third stage: numbers of people held. Node $j$ corresponds to demand state, $l$ is the work capability, $v$ is the initial capability and $s$ is the operation efficiency. 